\title{
Enjeux socio-cognitifs de l'engagement : la durabilité en contexte scolaire ${ }^{1}$
}

\section{Socio-cognitive issues of commitment: sustainability in school contexts}

\author{
Olivier Morin ${ }^{1}$, Jean-Marc Lange ${ }^{2}$, Angela Barthes ${ }^{3}$ \\ ${ }^{1}$ Université Claude Bernard de Lyon-1 (France), Laboratoire S2HEP, olivier.morin@univ-lyon1.fr \\ ${ }^{2}$ Université de Montpellier et Université Paul-Valery Montpellier (France), Laboratoire LIRDEF, \\ jean-marc.lange@umontpellier.fr \\ ${ }^{3}$ Aix-Marseille Université (France), ADEF, angela.barthes@univ-amu.fr
}

\begin{abstract}
RÉSUMÉ. Cette recherche s'inscrit dans la perspective d'une éducation au politique, émancipatrice, critique, créative et mobilisatrice permettant le dépassement des deux inhibitions fondamentales épistémique et sociale qui engendrent le doute voire le renoncement sur les possibilités individuelles ou collectives de transformer le monde dans une perspective de transition écologique. Cinq profils de l'engagement écocitoyen de jeunes arrivants en fin de scolarité obligatoire (14-17 ans) ont été établis au moyen d'une enquête par questionnaire $(n=170)$ actualisant et précisant ainsi les résultats obtenus lors d'une recherche précédente. Ces résultats sont ici complétés par la caractérisation sociologique et territoriale, mais aussi cognitive, tels les Raisonnements Socio Scientifiques dans la perspective de Durabilité, des cinq profils, au moyen de focus groupes. Sont pris en compte dans ce cadre et paramétrés, la dynamique des interactions, le rapport aux savoirs et au monde, dans ses dimensions identitaire, sociale et épistémique afin de déterminer des stratégies socio-didactiques plus efficientes car contextualisées.

ABSTRACT. This research follows the perspective of political, emancipatory, critical, creative, and engaging education allowing tto overcome two fundamental epistemic and social inhibitions which generate doubt or even renunciation of individual or collective possibilities to transform the world for an ecological transition. Five profiles of the eco-citizen engagement of young people arriving at the end of compulsory education (14-17 years) were established by means of a questionnaire survey $(n=170)$ updating and thus specifying the results obtained during a previous research. These results are now completed by the sociological and territorial characterization, but also cognitive dimensions, such as Socio Scientific Reasoning in the perspective of Sustainability, of the five profiles, by means of focus groups. The dynamics of interactions, the relationship to knowledge and to the World, in relation to identity, as well as social and epistemic dimensions, are taken into account in this framework and parameterized in order to determine contextualized and therefore more efficient socio-didactic strategies.

MOTS-CLÉS. Education au développement durable, scolarité obligatoire, engagement écocitoyen, Raisonnements Socio-scientifiques, stratégie socio-didactique.

KEYWORDS. Education for sustainable development, compulsory education, eco-citizen commitment, Socio-scientific reasoning, socio-didactic strategy.
\end{abstract}

L'éducation au développement durable est le plus souvent envisagée sous l'angle d'une sensibilisation des enfants, adolescents et jeunes à des enjeux généraux, visant l'adoption de comportement vertueux ou de «bonnes pratiques ». L'endoctrinement et la mise aux normes du monde de l'éducation aux idéologies dominantes et des lobbies techno-économicistes sont alors le plus souvent bien présents, quoique cachés (Barthes, 2018). D’une façon plus générale, l'éducation mise en œuvre sur les questions environnementales en contexte d'un anthropocène assumé se trouve en tension entre deux pôles : celui d'une éducation faible qui se contente de « sensibiliser » aux effets les plus néfastes d'un développement pensé essentiellement dans sa dimension technoéconomiciste ; celui d'une éducation forte qui vise la transformation sociétale au travers du développement de dispositions collectives et proactives (Lange, 2014). Dans le premier cas, les «

\footnotetext{
${ }^{1}$ Recherche effectuée dans le programme Education Interculturelle à l'Environnement et au Développement Durable (EIEDD, 2016-2020) soutenu par l'ANR en France et par le FRQSC au Québec.
} 
éducations à » sont mobilisées pour donner du sens à des apprentissages transversaux mais restent, en éducation formelle du moins, généralement incluses dans les disciplines existantes, dans un modèle d'autorité des experts et d'accumulation des savoirs - le « deficit model » des angloaméricains (Levinson, 2010). L'éducation forte vise plutôt la transformation sociétale au moyen d'une centration sur les enjeux/défis de l'anthropocène grâce à des projets participatifs territoriaux négociés et mobilisateurs (Lange et Martinand, 2010). Le projet est alors celui d'une éducation au politique, émancipatrice, critique, créative et mobilisatrice permettant le dépassement des deux inhibitions fondamentales à l'œuvre chez les jeunes d'âge scolaire : celle épistémique qui résulte de l'incommensurabilité de la tâche à accomplir, et celle sociale qui engendre le doute voire le renoncement sur les possibilités individuelles ou collectives de transformer le monde dans une perspective de transition écologique (Lange, Victor et Janner, 2011).

Le programme EIEDD (Education Interculturelle à l'Environnement et au Développement Durable ) s'inscrit dans cette dernière perspective. Il vise à établir des profils de l'engagement de jeunes arrivants en fin de scolarité obligatoire (14-17 ans) afin d'identifier les leviers curriculaires et les stratégies didactiques pertinents.

\section{La question de l'engagement des jeunes}

De nombreuses études, notamment nord-américaines mais également en Europe, en éducation aux sciences, à l'environnement et au développement durable, ont identifié une forme de fatalisme et de désengagement de la jeunesse face à la crise environnementale (Jenkins et Pell, 2006 ; Bader, Horman and Lapointe, 2010 ; Lange, 2012 ; Bader, Morin, Therriault, Arseneau, 2014 ; Zeyer et Kelzey, 2013). Les recherches rapportées dans plusieurs colloques internationaux ${ }^{2}$ ont précédemment établi que, pour mobiliser les jeunes à l'école et en tant que citoyens, il faut aussi tenir compte de leur identité, de leurs projets et relier davantage l'enseignement aux lieux qu'ils habitent. Dans un souci de développement durable (DD) des territoires et pour bien tenir compte des préoccupations des jeunes citoyens de cultures différentes qui y vivent, la recherche (EIEDD) en éducation interculturelle aux sciences, à l'environnement et au développement durable propose d'élaborer un cadre théorique franco-québécois afin de documenter comment des jeunes de 14 à 17 ans envisagent la durabilité d'un fleuve qu'ils côtoient et qui structure tant sur le plan paysager, qu'économique, environnemental et social le territoire où ils vivent. Pour ce faire, cinq recueils de données sont opérés dans des contextes culturels et environnementaux très différents. Ces données sont recueillies par questionnaires et entretiens de groupes en vue d'identifier et caractériser des " portraits types » de jeunes d'une part dans leurs rapports aux savoirs scientifiques et aux territoires, et d'autre part dans leurs dispositions et pratiques d'engagement écocitoyen eu égard aux enjeux sociaux, politiques, économiques, éthiques ou écologiques qu'ils associent au développement durable du fleuve en question. Deux terrains sont ciblés au Québec et trois en France. Au Québec et en France, un premier terrain est celui de jeunes d'un quartier multiculturel, alors que les autres sont ceux de jeunes d'un milieu plus rural dont la gouvernance est orientée par des principes de développement durable, afin de repérer des éléments culturels communs ou contrastés, plus locaux ou plus globaux. En France, les territoires jouxtent la Seine et au Québec, le Saint-Laurent. Cet article n'est relatif qu'au recueil de données français.Les établissements concernés sont un lycée proche de Paris (93), nous le nommerons LBP (Lycée en Banlieue parisienne), et deux autres situés dans ou en périphérie du Parc Naturel Régional «Boucle de la Seine Normande » (76) : un lycée

\footnotetext{
${ }^{2}$ À titre d'exemples, les colloques de : Digne (2010) Éducation au développement durable et à la biodiversité : concepts, questions vives, outils et pratiques, Rouen (2012) L'éducation au développement durable : appuis et obstacles à sa généralisation hors et dans l'Ecole, Clermont (2014) Éduquer et former au monde de demain. Place et rôles de la formation et de l'éducation, en vue d'une transition vers un monde durable, Montpellier (2018) Quel(s) curriculum(a) pour les Objectifs du Développement Durable?
} 
que nous nommerons LSR (Lycée Semi-Rural), et un collège que nous nommerons CRR (Collège Rural Riverain).

Les trois objectifs généraux de cette étude sont donc les suivants :

1) Dans le cadre général du concept de « rapport au savoir et au monde » (Charlot, 1997, Kalali, 2017) élaborer un cadre conceptuel et d'analyse original croisant les concepts de « rapport aux savoirs scientifiques», « rapport aux territoires» et «engagement écocitoyen» des jeunes de 14 à 16 ans scolarisés afin de comprendre dans quelle mesure une éducation forte ou faible est possible en contextes territoriaux et culturels différenciés.

2) Dégager des «portraits-types» de jeunes Québécois et de jeunes Français de 16 ans à l'égard du développement durable du Saint-Laurent au Québec et de la Seine en France, à partir de cinq études de cas.

3) Sur chaque territoire, en France et au Québec, avec les jeunes rencontrés en entretien, soutenir la conception collaborative de représentations interdisciplinaires d'un enjeu de développement durable et définir les formes d'engagement écocitoyen des jeunes selon les rapports aux savoirs scientifiques, les dimensions territoriales, identitaires et culturelles repérées.

Le présent article focalise sur ce troisième objectif.

\section{Méthodologie}

Partant d'un modèle socio-didactique de l'engagement, huit déterminants ont été mis à jour par une méthode statistique robuste vis-à-vis des enjeux de durabilité/soutenabilité permettant ainsi de caractériser cinq figures de l'engagement des jeunes vis-à-vis de ces enjeux : les figures sont : acteur/doute/paradoxal/spectateur réflexif/auteur (Lange et Barthes, 2020). Ils démontrent ainsi la permanence des processus d'inhibition « épistémiques » et « sociales » déjà identifiés en $2011^{3}$. Ils s'inscrivent en cela dans les caractérisations effectuées par Bandura (1986) et reprises dans les études internationales génériques sur l'engagement citoyen, celle du sentiment d'efficacité personnelle interne et le sentiment d'efficacité externe.

Afin de préciser les caractéristiques sociologiques et territoriales, mais aussi cognitives des élèves relevant de ces figures et donc d'envisager par la suite des stratégies socio-didactiques contextualisées et pertinentes à l'échelle individuelle et collective, les profils d'élèves précédemment évoqués sont croisés avec une analyse qualitative.

Ainsi, 19 entretiens de groupes de 3 à 5 élèves, correspondant à un corpus de 70 élèves au total, sont menés alors que ces derniers doivent suivre un protocole précis qui consiste à : 1- évaluer les enjeux de leur propre territoire 2- être confrontés à des productions d'autres établissements français ou québécois. Les verbatim sont récoltés et analysés, et des indicateurs construits, puis croisés aux caractéristiques socio-territoriales des élèves.

\footnotetext{
${ }^{3}$ Programme ED2AO, ANR-Blanc 2008-2011 


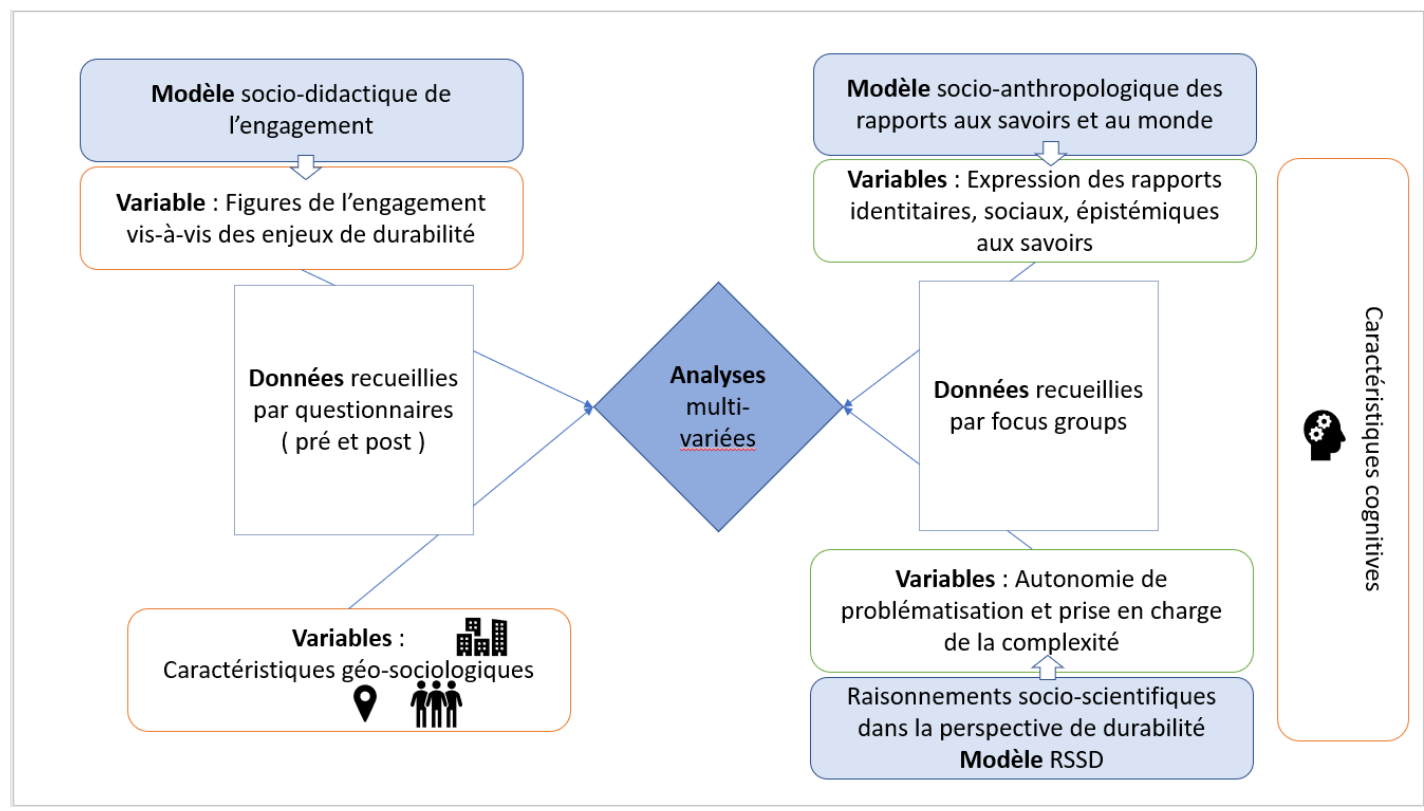

Figure 1. Croisement des figures d'engagement des jeunes vis-à-vis des enjeux de durabilité avec les caractéristiques sociologiques, territoriales et cognitives analysées

\subsection{Mise en place d'un dispositif pédagogique permettant de discuter des enjeux de durabilité du fleuve structurant le paysage des établissements scolaires}

La phase du projet EIEDD qui nous intéresse ici s'est déroulée en 2018/2019, année scolaire au cours de laquelle les projets pédagogiques interdisciplinaires dans les cinq établissements visaient des échanges interculturels. Dans chacun des établissements partenaires, les groupes d'élèves ont été accompagnés dans l'élaboration d'une représentation interdisciplinaire d'un enjeu de durabilité ${ }^{4}$ lié au fleuve qu'ils côtoient. L'estimation du volume horaire dédié au projet par les classes a été d'environ $30 \mathrm{H}$ en présentiel : dans le cadre d'un «club » au collège, de séances d'accompagnement personnalisé conduites par des enseignants de Sciences de la vie et de la Terre (SVT) et Histoire et géographie $(\mathrm{HG})$ en lycée. Le scénario pédagogique a été co-construit avec les enseignants selon une alternance de phases (Présenter/Échanger/Rendre compte) dans le but de relier une mise en dynamique à partir de constats locaux à une mise en perspective autour d'enjeux globaux. La présentation de la démarche et le document synoptique partagé sur la plateforme collaborative sont rapportés en annexe $\mathrm{n}^{\circ} 1$.

\subsection{Focus groupes, principes et organisation}

La méthode des groupes centrés («focus groupes ») est une méthode de recherche fondée sur la communication de groupe, particulièrement indiquée en perspective comparative (Bugnard, Freudiger, Haeberli, \& Pache, 2009). Il s'agit de mobiliser des groupes dans des discussions focalisées sur l'analyse et l'évaluation de solutions envisagées face à un problème posé. Ces communications socialement situées favorisent l'expression de la diversité d'opinions ainsi que la négociation et la création de nouvelles significations partagées (Marková, 2003). L'interaction entre les participants est explicitement ciblée (Kitzinger, Marková, \& Kalampalikis, 2004). En stimulant la prise de position de l'individu par rapport au groupe, les échanges sont de nature à permettre de mieux saisir les motivations et les éléments qui sous-tendent les rapports à un objet que dans un entretien individuel.

\footnotetext{
${ }^{4}$ D'après les réponses aux questionnaires de l'étude préliminaire, nous savions au début de cette phase du projet que les élèves de ces établissements associent au fleuve des enjeu(x) de durabilité à propos des questions de patrimoine, d'espace de biodiversité, de symbole culturel, d'espace public et de bien-être, de voie de transport.
} 
Les focus groupes doivent permettre aux participants de penser à voix haute et à plusieurs. Dans notre étude, des groupes de 3 à 5 élèves sont conduits à interroger et remodeler leurs opinionsraisonnées (Lange, Trouvé, \& Victor, 2007) sur les relations entre les activités humaines et la biodiversité d'un fleuve, via deux confrontations à la production de groupes d'élèves d'un autre établissement français ou québécois. A partir de ces deux documents, il s'agit de permettre aux élèves d'exprimer ce qui leur paraît intéressant, étonnant, dérangeant, intrigant, etc. Nous analysons les expressions de leur rapport aux savoirs et au monde, ainsi que la dynamique des interactions durant trois temps d'échanges. Précisons ces moments :

Le temps 1 des entrevues conduit les élèves à exprimer leur ressenti de la démarche de projet vécue autour des enjeux de durabilité du fleuve ${ }^{5}$. Le temps 2 est celui de leurs réactions au document $1^{6}$ : diaporama produit par un groupe du CRR travaillant sur les populations d'animaux aquatiques de la Seine. Le temps 3 est celui de leurs réactions au document $2^{7}$ : un site web «qui traite de l'état du Saint-Laurent, des facteurs qui lui sont nuisibles ainsi que des lois actuellement en vigueur afin d'éviter la dégradation de cet important cours d'eau » recommandé à tous les participants via la plateforme collaborative par un élève de Québec.

\subsection{Construction des indicateurs sur les entretiens, analyse des verbatim}

\subsubsection{Caractéristiques géo-sociologiques}

Les trois établissements français désignés respectivement LBP (Lycée en Banlieue parisienne), LSR (Lycée Semi-Rural), et CRR (Collège Rural Riverain) ont été sélectionnés d'une part par leur situation géographique de proximité relative par rapport au fleuve mais aussi par leur diversité sociologique et culturelle : sont pris en compte les Catégories Socio Professionnelles (CSP) des parents (et en particulier de la mère), le type d'établissement (LBP labellisé E3D ; CRR comportant un club DD), la diversité culturelle (jeunes de banlieue dite sensible, très majoritairement issus de l'immigration récente ; jeunes ruraux ou néoruraux, majoritairement de « souche ancienne » ...).

Sont alors pris en compte dans ce cadre et paramétrés la dynamique des interactions durant les focus-groupes et le rapport aux savoirs et au monde exprimé par les élèves durant les échanges. Cette caractérisation fine des profils sociologiques, territoriaux et cognitifs des élèves permet ainsi d'établir une cartographie des dynamiques d'apprentissages et d'engagement.

\subsubsection{Caractéristiques cognitives}

\section{Expression des rapports aux savoirs et au monde}

Le modèle des rapports aux savoirs (Charlot, ibid.) conduit à explorer l'articulation des parcours singuliers et des rapports sociaux. Ce travail est souvent opéré afin de comprendre les situations scolaires de réussite, d'échec, voire de décrochage scolaire. Il s'agit donc dans l'étude des rapports aux savoirs de comprendre les articulations d'une signification sociale (en général en lien avec un but) avec un sens plus individuel. Les rapports sociaux se construisent dans une histoire collective et dans une relation personnelle à ces modèles sociaux. Par ailleurs, considérer les rapports aux savoirs implique d'appréhender l'activité de leur appropriation. Dans l'éducation, il s'agit d'un rapport à un processus d'apprentissage. Plus largement, c'est le fait de posséder la maîtrise d'une activité de réflexion en situation. Ainsi donc, selon le champ socio-anthropologique des rapports aux savoirs, trois dimensions sont à considérer : la relation de sens du sujet vis-à-vis de son objet de savoir ; la relation aux modèles sociaux ; la relation au processus d'appropriation. Dans le vocable des rapports

\footnotetext{
${ }^{5}$ Le guide d'entretien de cette première partie est en annexe $n^{\circ} 2$

${ }^{6} \mathrm{Cf}$. annexe 3

${ }^{7}$ Cf. annexe 4
} 
aux savoirs, il s'agit respectivement des dimensions dites identitaires ; sociales ; épistémiques. Nous avons développé une méthode pour approcher et positionner dans un ensemble reproductif les dimensions des rapports aux savoirs, dans laquelle chacune de ces trois dimensions ou variables définit des facteurs d'analyses susceptibles d'être quantifiés. Pour chaque variable, des indicateurs permettent de rapporter une situation de terrain observée à l'une des modalités statistiques de celleci c'est-à-dire qu'un score lui est attribué (de 1 à 5 ; tableau 1, ci-dessous). Pour chaque focus groupe, l'individu considéré est la triade de participants.

\begin{tabular}{|c|c|c|c|}
\hline Modalités & $\begin{array}{l}\text { Variable } 1 \text {, dimension } \\
\text { identitaire }\end{array}$ & Variable 2, dimension sociale & Variable 3, dimension épistémique \\
\hline $\begin{array}{c}\text { modalité } \\
1\end{array}$ & $\begin{array}{l}\text { La problématisation est } \\
\text { déconnectée d'investissement } \\
\text { identitaire. }\end{array}$ & $\begin{array}{l}\text { Les questions de normes ne } \\
\text { sont pas abordées. }\end{array}$ & $\begin{array}{l}\text { Les apports d'informations nouvelles ne } \\
\text { sont pas identifiés. }\end{array}$ \\
\hline $\begin{array}{l}\text { modalité } \\
2\end{array}$ & $\begin{array}{l}\text { Les participants expriment une } \\
\text { facette de leur identité propre. }\end{array}$ & $\begin{array}{l}\text { Les participants indiquent « ce } \\
\text { qui se fait », ce qu'il } \\
\text { « convient de faire ». }\end{array}$ & $\begin{array}{l}\text { Les participants constatent eux-mêmes } \\
\text { des différences dans les informations } \\
\text { mobilisées, sans modifier pour autant } \\
\text { leur raisonnement. }\end{array}$ \\
\hline $\begin{array}{c}\text { modalité } \\
\mathbf{3}\end{array}$ & $\begin{array}{l}\text { Les participants mobilisent } \\
\text { une/des facettes de leur identité } \\
\text { propre dans leur } \\
\text { problématisation. }\end{array}$ & $\begin{array}{l}\text { Les participants soulignent des } \\
\text { différences sur ce qui paraît } \\
\text { «normal » à différents } \\
\text { groupes. }\end{array}$ & $\begin{array}{l}\text { Les participants intègrent des } \\
\text { connaissances nouvelles dans leur îlot } \\
\text { de rationalités (sans les remettre en } \\
\text { question). }\end{array}$ \\
\hline $\begin{array}{c}\text { modalité } \\
4\end{array}$ & $\begin{array}{l}\text { Les participants mobilisent dans } \\
\text { leur problématisation des facettes } \\
\text { de leur identité propre ET de } \\
\text { l'identité du territoire dans lequel } \\
\text { ils habitent. }\end{array}$ & $\begin{array}{l}\text { Les participants s'interrogent } \\
\text { sur ce qui paraît « normal » } \\
\text { dans leurs différents contextes. }\end{array}$ & $\begin{array}{l}\text { Les participants questionnent la fiabilité } \\
\text { des différentes informations reçues. }\end{array}$ \\
\hline $\begin{array}{c}\text { modalité } \\
5\end{array}$ & $\begin{array}{l}\text { Les participants justifient leur } \\
\text { problématisation par des facettes } \\
\text { de leur identité propre et/ou de } \\
\text { l'identité du territoire dans lequel } \\
\text { ils habitent. }\end{array}$ & $\begin{array}{l}\text { Les participants discutent (et } \\
\text { donc explicitent) leur } \\
\text { conformité aux usages, } \\
\text { traditions et pratiques de leurs } \\
\text { contextes socio-culturels. }\end{array}$ & $\begin{array}{l}\text { Les participants discutent (et donc } \\
\text { explicitent) les intérêts et limites, la } \\
\text { cohérence ou la pertinence des diverses } \\
\text { connaissances mobilisées dans les } \\
\text { documents } 1 \text { et } 2 \text {. }\end{array}$ \\
\hline
\end{tabular}

\section{Tableau 1. Indicateurs de l'épistémologie des rapports aux savoirs exprimée en variables et modalités statistiques}

\section{Autonomie de problématisation}

La dynamique des interactions durant les focus-groupes, en particulier entre l'animateur et les participants, est retenue pour capter le niveau d'autonomie des groupes dans la problématisation (au sens de Fabre, 2017) des enjeux de durabilité. Lorsque les échanges portent sur le document 1, puis sur le document 2, nous dénombrons les prises de parole d'élèves en réponses directes à une question de l'animateur d'une part, le nombre de prises de parole entre élèves dans des échanges dialogués sans intervention de l'animateur d'autre part. L'autonomie de problématisation est alors quantifiée par le calcul du rapport entre ces deux mesures : nombre de réponses à un autre élève/nombre de réponses à l'animateur. 
Le premier extrait présenté en 4.3 rapporte par exemple 7 prises de parole d'élèves, dont 3 sont des réponses directes à l'animateur, et 4 des réponses à un autre élève, donc un quotient d'autonomie de problématisation, pour cet extrait, de 4/3. Il est de 0 pour le deuxième extrait rapporté en 4.3 où les 22 prises de parole d'élèves sont toutes en réponse à l'animateur, et de 3/12 pour le troisième extrait rapporté en 4.3. Précisons que les mesures utilisées dans les analyses statistiques de la partie résultats portent sur l'ensemble des temps d'échanges et non sur les seuls extraits cités dans cet article.

\section{Prise en charge de la complexité}

Concernant les caractéristiques cognitives, ce sont les «Raisonnements Socio Scientifiques dans la perspective de durabilité » (RSSD, Morin, 2013) qui sont paramétrés, notamment leur complexité. «Un objet complexe étant un nœud dans un réseau, l'appréhension d'un problème complexe passe par la prise en compte du contexte en tant que réseau d'interactions. L'approche de la complexité ne focalise donc pas la réflexion sur un simple facteur en excluant sa signification dans un contexte large, et ne se base pas sur une simple relation de causalité, mais au contraire reconnait les multiples interactions dynamiques entre les systèmes considérés. » (Morin, 2017, p 66).

La prise en charge de la complexité par les groupes aux moments où leur sont soumises les productions d'autres groupes est captée en première approche par le nombre de liens explicités par les participants entre les items du raisonnement en construction ${ }^{8}$. Le nombre (n) d'items mobilisés dans les raisonnements et le nombre (l) de liens est mesuré i) dans leur propre production (11/n1) telle qu'ils l'ont publiée dans la plateforme collaborative, ii) lorsqu'ils intègrent à leur raisonnement les apports du document 1 pendant le focus groupe $(12 / \mathrm{n} 2)$, puis iii) lorsqu'ils intègrent à leur raisonnement, toujours pendant le focus group, les apports du document 2 (13/n3). Ces analyses s'appuient sur la traduction des raisonnements dans des cartes mentales telle que celle présentée en annexe $n^{\circ} 5$. Pour le groupe étudié dans cet exemple ${ }^{9}$, le rapport $11 / \mathrm{n} 1=2 / 3$ (éléments en jaune), $12 / \mathrm{n} 2=9 / 11$ (éléments en jaune et en orange), et $13 / \mathrm{n} 3=15 / 16$ (éléments en jaune, en orange et en vert)

\subsection{Croisement des données élèves, groupes, contextes}

Nos analyses visent à préciser les caractéristiques sociologiques et territoriales, mais aussi cognitives relevant des profils de l'engagement de ces jeunes arrivant en fin de scolarité obligatoire (14-17 ans). Puisqu'il s'agit de prendre en compte simultanément plus de deux variables, nous mobilisons les outils d'analyses en composantes multiples (Larini et Barthes, 2018; Barthes et Larini, 2020). Si les bases mathématiques des analyses multivariées sont complexes (Benzecri, 1973), la simplicité de leur mise en œuvre avec un logiciel adapté ${ }^{10}$ est avérée mais avec quelques précautions, et les potentialités d'adaptations aux sciences de l'éducation infinies.

Le principe est de considérer les variables sans a priori sur leurs corrélations, et de donner du sens au corpus en décrivant les répartitions qui s'écartent de celle - uniforme - qui traduirait l'indépendance entre les variables. Nous utilisons 1'Analyse Factorielle de Correspondance (AFC) pour les variables catégorielles (établissement, genre, CSP de la mère, type d'engagement, rapport identitaire, rapport social, rapport épistémique aux savoirs et au monde) et l'Analyse en Composantes Principales (ACP) pour les variables numériques (autonomie de problématisation avec le document 1 puis avec le document 2, indice de prise en charge de la complexité dans la

\footnotetext{
${ }^{8}$ Dans une seconde approche, que nous rapporterons dans un autre article, la nature qualitative de ces mises en lien est analysée.

9 II s'agit des élèves cités dans le troisième extrait rapporté en 4.3

${ }^{10}$ Pour information à titre d'exemple : Xcel Stat, SPHINX, SPSS, Modalisa, SAS et R.

(C) 2021 ISTE OpenScience - Published by ISTE Ltd. London, UK - openscience.fr 
préparation - c'est-à-dire lors de la production publiée par le groupe dans la plateforme collaborative- puis avec le document 1 , et avec le document 2).

\subsection{Les profils de modalités}

Comme avec les analyses multivariées, l'hypothèse fondant l'approche par modalités est que des variables peuvent être mises en relation, mais sans présupposer desquelles il s'agit. Cette fois, la démarche consiste à regrouper à partir de la matrice de données ${ }^{11}$ les individus partageant la même valeur d'une variable puis de rechercher les valeurs des autres variables qui regroupent le plus les mêmes individus.

La figure 2 ci-dessous est une capture d'écran d'un exemple de requête de profil de modalité (logiciel Modalisa).

\begin{tabular}{|c|c|c|c|c|c|c|}
\hline \multicolumn{7}{|c|}{$\begin{array}{l}\text { 9. rapport à. Social } \\
\text { Modalité } 2 \text {. différences de normes sociales soulignées selon les contextes ( } 32=57,1 \%)\end{array}$} \\
\hline Variable & Modalité & Effectifs & Ecarts & s Khi2 & PEM & $\begin{array}{l}\text { Test } \\
\text { Khi2 } \\
\text { local }\end{array}$ \\
\hline 4. genre & Fllles & 24 & & $\begin{array}{ll}6 & 1,786\end{array}$ & $42 \%$ & ... \\
\hline 8. rapport à. Identitaire & 4 & 17 & & $7 \quad 5,464$ & & $\ldots$ \\
\hline
\end{tabular}

Figure 2. Exemple d'un profil sur une modalité NB : l'intitulé de la modalité 2 a été simplifié ici, l'indicateur complet figure dans le tableau 1

Ainsi, pour la variable « rapport au social », modalité 2 il y a 32 individus, représentant 57,1\% de l'échantillon. Le logiciel n'affiche que les modalités des autres variables qui lui sont significativement liées. Dans cet exemple, le rapport au social et sa modalité « différences de normes sociales soulignées selon les contextes, est liée avec la variable « genre » pour 24 des 32 individus qui sont des filles, et avec la variable « rapport à identitaire » pour 17 individus ayant exprimés la modalité 4 , les $\chi^{2}$ et pourcentages d'écarts à la moyenne (PEM) à l'appui.

Nous verrons dans la partie « résultats » que ces analyses permettent à la fois de confirmer les relations identifiées avec les analyses multivariées (en retrouvant les variables liées) et de préciser leurs interprétations (en lisant les modalités associées).

\footnotetext{
${ }^{11}$ Cette matrice de données est le tableau qui regroupe les infos pour les $\mathrm{n}$ variables et les $\mathrm{x}$ individus. 


\section{Résultats}

\subsection{Les dimensions des rapports aux savoirs déterminantes}

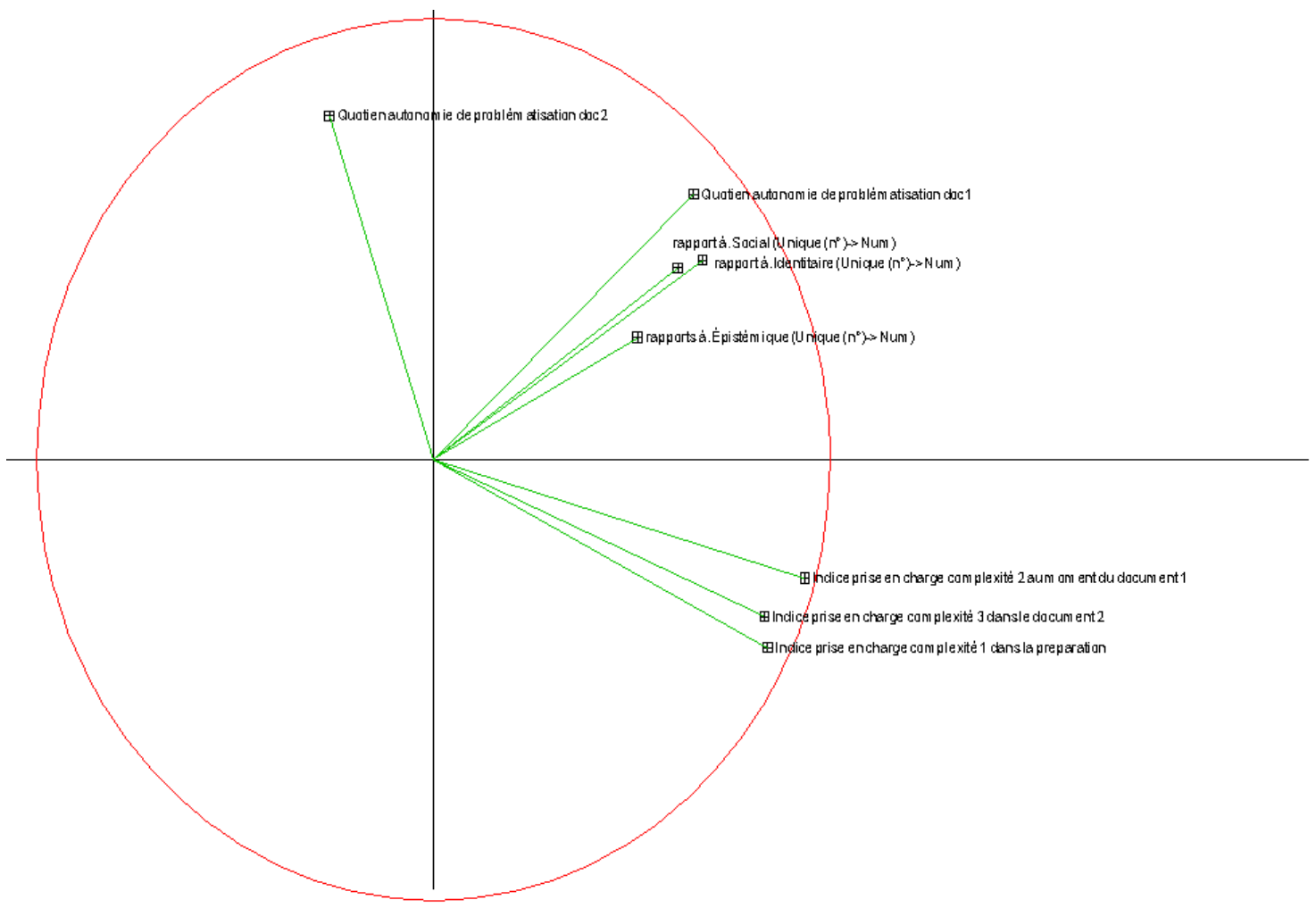

Figure 3. ACP des variables de rapports aux savoirs, d'autonomie et de prise en charge de la complexité $\%$ Information

Facteur $1: 49,61 \%$

Facteur $2: 23,79 \%$

\begin{tabular}{|c|c|c|}
\hline Variables & Facteur 1 & Facteur 2 \\
\hline V1 : rapport à. Identitaire & 0,677 & 0,452 \\
\hline V2 : rapport à. Social & 0,616 & 0,434 \\
\hline V3 : rapports à. Épistémique & 0,513 & 0,276 \\
\hline V4 : Quotient autonomie de problématisation doc 1 & 0,654 & 0,603 \\
\hline V5 : Quotient autonomie de problématisation doc 2 & $-0,261$ & 0,780 \\
\hline V6 : Indice prise en charge complexité 1 dans la préparation & 0,842 & $-0,427$ \\
\hline V7 : Indice prise en charge complexité 2 au moment du document $\mathbf{1}$ & 0,935 & $-0,267$ \\
\hline V8 : Indice prise en charge complexité 3 dans le document $\mathbf{2}$ & 0,834 & $-0,355$ \\
\hline
\end{tabular}

Tableau 2. Variables factorielles en fonction des variables initiales et \% information cumulée par chacun des axes factoriels de l'ACP 
Le sens des "vecteurs variables" nous renseigne sur la signification des 4 cadrans. Nous n'entrons pas ici dans les développements mathématiques conduisant à cette représentation. Retenons que la valeur de la variable factorielle pour chacun des axes détermine la longueur des « vecteurs variables »; ainsi, dans le cas général au plus les extrémités sont éloignées du cercle de rayon 1, au moins l'ACP est de bonne qualité. Retenons également que lorsque deux variables sont très corrélées elles font entre elles un angle voisin de 0 si le coefficient de corrélation est positif (ici par exemple l'expression du rapport social et l'expression du rapport identitaire aux savoirs et au monde) ou voisin de $180^{\circ}$ s'il est négatif (ici par exemple l'indice de prise en charge de la complexité avec le document 2 et l'autonomie de problématisation avec le même document). Si deux variables sont peu corrélées, elles font entre elles un angle important pouvant aller jusqu'à $90^{\circ}$ (par exemple ici l'autonomie de problématisation avec le document 1 et celle avec le document 2).

L'ACP réalisée est de très bonne qualité, car le plan principal contient $49,61 \%+23,79 \%=73,40$ $\%$ des informations du tableau de données initial. Les 8 variables sont bien représentées à l'intérieur du cercle des corrélations, car leur extrémité est proche du cercle. Les angles que font les 8 vecteurs variables montrent que V1 (dimension identitaire) et V2 (dimension sociale) sont très corrélées. En revanche V3 (dimension épistémique) est peu corrélée avec V1 et V2, et V4 (le quotient d'autonomie de problématisation) est partiellement corrélées aux trois autres variables. Il est possible d'en conclure que les dimensions des rapports aux savoirs sont fondamentales aux sujets pour problématiser des enjeux de développement durable. La contribution des variables initiales aux variables factorielles permet de donner du sens à chacune des zones du plan.

Plus le point se déplace vers la droite et plus les 3 dimensions du rapport aux savoirs (V1 + V2 + V3) sont importantes, de même que pour V4. Ainsi, plus les individus sont positionnés à droite et plus ils sont favorablement influencés par ces 4 variables. Plus ils sont positionnés à gauche et moins ils sont influencés. Du point de vue de l'étude de cas, les sujets qui sont les moins autonomes dans la problématisation des enjeux de développement durable de leur territoire de proximité sont ceux qui ont peu d'investissement identitaire et qui interrogent peu les normes. D'un point de vue éducatif, conduire les élèves à interroger les normes, en particulier les normes dominantes d'un point de vue critique permet une prise en charge citoyenne des enjeux de développement.

Plus les individus sont positionnés haut dans le plan, plus ils discutent et intègrent les nouvelles informations, et plus a priori ils sont à même de problématiser les enjeux, même si la variable V4 (autonomie de problématisation) n'est que plus modérément corrélée.

L'examen des variables qualitatives des individus positionnés dans le cercle montre donc que ce ne sont pas spécialement les élèves les plus performants, ou ceux dont les parents sont CSP + qui comprennent le mieux les enjeux de durabilité, mais ceux dont les rapports aux savoirs se trouvent dans les modalités 4 et 5 , donc ceux qui expriment une identité propre et celle de leur territoire en même temps qu'ils discutent les normes. La variable genre n'intervient pas ici. En réalité, les milieux urbains sensibles et les milieux ruraux isolés sont les plus autonomes dans la problématisation, ce qui renvoie bien, sans rentrer dans les détails, à l'adaptation indispensable des enseignements aux contextes entre autres.

\subsection{Trois modes d'appréhension des enjeux de durabilité révélés par l'AFC}

Dans la figure 4 ci-dessous, les positionnements des différentes catégories de la variable « rapport identitaire au monde » sont indiqués en vert, ceux du « rapport social au monde » en violet, ceux du « rapport épistémique au monde » en rose, les types d'engagement avant la démarche en violet et après la démarche en rouge. 


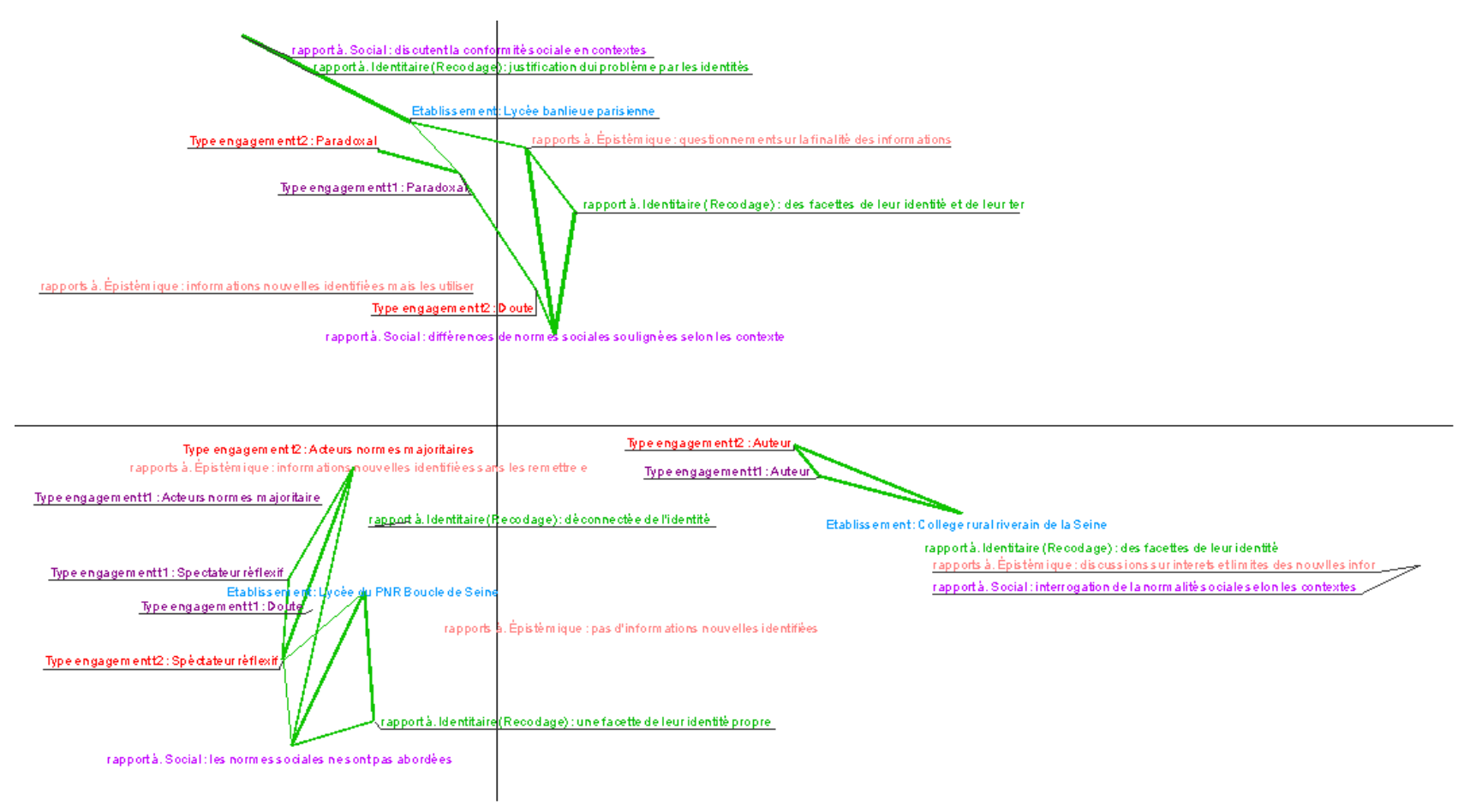

Figure 4. AFC liant établissements, types d'engagement, et rapports aux savoirs $\%$ Information

Facteur $1: 48,88 \%$

Facteur $2: 25,321 \%$

La conservation de l'information initiale dans cette AFC est de $74 \%(48,88+25,321=74,201)$ ce qui est statistiquement satisfaisant et autorise à donner une signification concrète aux axes horizontaux et verticaux correspondants respectivement aux facteurs 1 et 2 . Les modalités et variables consignées dans la figure 4 sont donc significatives. De bas en haut, nous observons que la répartition des catégories de variables est organisée selon un gradient de prise en compte des normes et des contextes sociaux, ce qui tend à donner à l'axe vertical le sens de la problématisation sociale des enjeux. La répartition des catégories de variables de gauche à droite traduit un gradient de diversité de prise en compte des informations nouvelles dans les raisonnements, ce qui conduit à donner cette signification à l'axe horizontal.

Cette AFC met en évidence plusieurs relations entre les expressions des rapports aux savoirs et au monde d'une part et des profils de l'engagement des élèves d'autre part. Nous observons en effet dans la figure 4 :

- au-dessus de l'axe horizontal, un ensemble de modalités de variables d'expression des rapports aux savoirs et au monde marqué par la mise en avant des appartenances sociales et territoriales, associé à deux profils d'engagement en fin de démarche : le type «paradoxal » et le type « doute »

- en bas à gauche, un ensemble de modalités de variables d'expression des rapports aux savoirs et au monde marqué au contraire par l'expression neutre des problématiques de durabilité prises comme des exercices scolaires, associé aux figures d'engagement en fin de démarche du type " spectateur réflexif » et « acteur»

- en bas à droite, des modalités de variables d'expression des rapports aux savoirs et au monde marquées par le questionnement des élèves sur les informations nouvellement reçues, qu'elles soient de l'ordre des différences sociales, identitaires ou épistémiques (cf. 3ième verbatim, § 4.3), associé au profil d'engagement « auteur » en fin de démarche.

L'AFC montre également une relation entre ces variables et des caractéristiques sociologiques et territoriales. Nous constatons que : 
- le premier ensemble correspond à un mode de problématisation des enjeux de durabilité surtout observé parmi les élèves du Lycée de Banlieue parisienne (LBP), établissement regroupant des élèves dont l'activité socio-professionnelle de la mère (cf. § 3.3.1) est majoritairement dans la catégorie « employé »

- le deuxième à un mode de problématisation des enjeux de durabilité surtout observé parmi les élèves du Lycée Semi-Rural (LSR), établissement regroupant des élèves dont l'activité socioprofessionnelle de la mère est majoritairement dans la catégorie «profession intermédiaire »

- et le troisième à un mode de problématisation des enjeux de durabilité surtout observé parmi les élèves du Collège Rural dans la Réserve naturelle (CRR), établissement regroupant des élèves dont l'activité socio-professionnelle de la mère est majoritairement dans la catégorie « cadres et prof. int. sup».

Ainsi, le croisement des analyses des réponses individuelles au questionnaire d'une part avec l'expression des rapports aux savoirs et au monde durant les entretiens collectifs d'autre part permet d'identifier des caractéristiques cognitives et sociales de différents profils d'engagements vis-à-vis des enjeux de durabilité. Cela se traduit dans la figure 4 par la répartition des modalités de variables en trois ensembles distincts, au sein desquels les traits épais correspondent aux fortes valeurs des mesures du test $\times 2$ de contingence.

De plus, nous avons vu que l'axe vertical dans le plan des composantes principales correspond à un gradient de prise en compte des normes et contextes sociaux. On observe, et c'est un effet positif du dispositif pédagogique, que pour chacun des profils d'engagement il y a un décalage vers le haut entre « l'avant » et «l'après » de la démarche, révélant que ces jeunes prennent davantage en considération la dimension sociale de la problématisation suite au projet mené durant l'année. Celuici leur a permis d'élargir leur appréhension des enjeux de durabilité au-delà des aspects environnementaux au sens biophysique du terme.

\subsection{Zooms sur ces modes d'appréhension des enjeux de durabilité}

Les analyses « de profil par modalités » permettent de retrouver les traits caractéristiques identifiés par l'AFC ci-dessus, et de préciser leur interprétation. Nous allons ci-dessous les présenter. Pour compléter ces analyses qui portent sur des individus statistiques et en retrouvant la dimension humaine de cette étude qui porte avant tout sur des personnes ${ }^{12}$ sociales. Ainsi, nous citerons quelques verbatim propres à rapporter leur mode d'appréhension des enjeux de durabilité (nous avons passé en bleu dans ces verbatims les éléments sur lesquels nous souhaitons porter l'emphase).

\subsection{1. «Paradoxaux»}

L'analyse par modalités révèle que les 17 individus exprimant leur identité propre ET leur identité territoriale durant l'entretien (focus-groupe) sont aussi ceux qui soulignent les différences de normes sociales selon les contextes. Ces jeunes correspondent au profil «paradoxaux » dans l'ensemble de relations repéré dans la partie supérieure de la figure 4. Ils expriment à la fois un appel à la critique sociale et à l'action politique en faveur du respect de l'environnement, et un sentiment d'impuissance, de désabusement vis-à-vis de leur pouvoir d'agir. Considérant que les problèmes environnementaux leur sont externes et nécessitent la punition des responsables, ils les

\footnotetext{
${ }^{12}$ Selon l'étymologie traditionnelle, « personne » vient du latin persona [...]. Persona, qui était le masque de scène, est devenu peu à peu le porteur de masque, l'acteur, puis le personnage joué par l'acteur, le rôle.

https://www.universalis.fr/encyclopedie/personne/1-de-l-etymologie-a-la-metaphysique/ consulté le 16 juin 2020

(c) 2021 ISTE OpenScience - Published by ISTE Ltd. London, UK - openscience.fr

Page | D-12
} 
dénoncent tout en appelant à des mesures coercitives et en assumant la légitimité de leur nonimplication dans la recherche de solutions :

[...] V - Dans le 93, donc banlieue -et encore, ce n'est pas une banlieue très éloignée parce que on dépasse le pont et on est à Paris ; on est même pas à $1 \mathrm{~km}$ de Paris, et même, enfin en fait, Paris déjà y'a... c'est recentré là où c'est beau en fait c'est que dans les beaux quartiers donc les Champs-Élysées, etc ; alors que ici, c'est sale.

Là tu dis que en fait la Seine elle est plus polluée, où ? Autour de la Seine, c'est plus pollué ici que...

V-ben oui !

... Que à Paris ?

$\mathrm{S}$ - ben clairement ! [tout le monde parle en même temps]

V - Ceux de [CRR] où je sais pas quoi là ; si ils ont l'occasion un jour d'aller à Paris, et, ensuite, de venir ici, ils verront la différence ! Nous, ici, la Seine, mais clairement, moi je vous le dis : si vous voulez y aller, allez-y ! Moi j'y vais des fois, vous arrivez, des arbres partout, aucun escalier y'a même des sortes de minis euh des minis... je sais pas comment dire... des minis... des sortes de... là où les gens ils vivent, y'a des... tu vois... tu vois que y'a des bêtes partout, ça pue, y'a des objets et tout ! Tu vas à la Seine vers Notre-Dame, alors ça sent bon, c'est propre, tu peux te balader, y'a des jeux à côté, y'a des jeux avec de l'eau... enfin c'est... c'est normal que nous, on a moins envie de prendre soin de la Seine ! On voit que la Seine elle est déjà délaissée par 1'État, c'est pas nous 400,000 enfin je sais pas, à XXX on est 40,000 personnes, euh c'est pas à nous 40,000 habitants, de banlieue, c'est pas à nous de prendre soin de la Seine. $[\ldots]$

[...] qu'est-ce qu'on pourrait proposer comme action pour changer, pour aller plus... Pour favoriser le développement durable de la Seine ? Oui ?

L - alors je vais être assez claire sur le sujet, - alors euh... quand les campagnes de prévention qui ont été mises en place pour la Seine, n'ont clairement pas fonctionné, on retrouve toujours des déchets, des débris de gas-oil, ou encore de scooters, des déchets plastiques, ou encore plein de choses. Donc en fait les gens ne prennent pas vraiment au sérieux l'importance de la Seine, donc je pense qu'il faudrait plutôt prendre de manière assez radicales donc... des amendes, ou des...

X\# ça ça va être mis en place.

L - ... ou d'autres choses comme ça, donc qui va faire intervenir par exemple les forces de l'ordre ou l'État parce que c'est un sujet qui nous concerne tous et on est tous impliqués làdedans. $[\ldots]$

\subsection{2. «Spectateurs réflexifs » et « acteurs »}

Une autre analyse par modalités permet d'identifier 17 autres élèves, dont l'autonomie de problématisation est réduite. Ce sont des élèves en difficulté pour appréhender sans aide les problématiques de durabilité. Parmi eux, 15 ne prennent en charge que moyennement la complexité 
globale. 10 mobilisent peu leur identité dans la problématisation des enjeux . Leur profil se rapporte aux types «spectateurs réflexifs » et « acteurs » de l'ensemble de relations identifié en bas à gauche de la figure 4. L'extrait suivant illustre un échange avec de tels élèves :

[...] Alors à votre avis, est-ce que l'état de la Seine et l'état du Saint-Laurent, c'est la même chose ? Ou y'a un fleuve qui irait bien et...

E : Non, parce que c'est un peu les mêmes usines partout. Enfin... pas les mêmes usines, mais ils rejettent les mêmes produits, donc si le Saint-Laurent va mal, la Seine doit être aussi un peu pareil.

Ouais. Ils disent aussi, dans ce document, que la nature de la pollution a changé. Alors pourquoi ça a changé ?

E : Parce qu'on a interdit des produits mais y'en a d'autres qui sont arrivés.

Oui. Et ils arrivent comment?

$\mathrm{E}$ : Ben à cause de nous. Par les usines, par les bateaux.

Les bateaux ? Ok. Donc pour le Saint-Laurent, on vous explique aussi qu'il y a eu un plan d'action, qui s'appelle Saint-Laurent vision 2000, pour établir l'état de santé, on va dire, du fleuve. Est-ce que vous savez s'il y a eu quelque chose d'équivalent pour la Seine ?

E : Non

\section{A : Pas du tout.}

Alors si la question des pollutions, la nature des pollutions a changé, qu'est-ce qu'on peut faire en fait?

\section{Long silence}

E : Si la nature des pollutions a...

A changé - c'est ce qui est dit hein dans, dans ce texte :

E : oui

il y avait des pollutions qui étaient connues -

E : oui

les rejets industriels etc. Il y a eu un plan, un plan d'action pour hé bien mieux contrôler les rejets de l'industrie, mais aussi des villes, etc, le long du Saint-Laurent. En fait, y'a eu la même chose hein le long de la Seine, sauf que depuis, on a trouvé qu'il y avait d'autres problèmes. Alors est-ce que les solutions qui étaient proposées par les élèves de [CRR] sont valables dans ce contexte-là ?

E : Ben non, moins. 
Expliquez-un peu pourquoi vous dites ça ?

A : Parce que là, la Seine elle est polluée, donc on peut pas dépolluer... en faisant la pêche électrique.

Qu'est-ce qui est pollué, en fait ?
A : L'eau.

E : Les fonds.

L'eau ou les fonds, les sédiments?

E : Ben les fonds, plus. Parce que la pollution, elle retombe dans le fond

Alors qu'est-ce qu'on peut faire, si c'est les sédiments qui sont pollués ? Qu'est-ce que vous proposeriez?

$\mathrm{E}:$ Ben... comme ils avaient dit, faire des drainages, pour remettre les sédiments à la surface et après les reprendre. Si c'est possible [sourire].

Est-ce que c'est possible, ça ? De reprendre tous les sédiments de la Seine et... et puis on en fait quoi ?

A : Ben oui, c'est ça : parce qu'après, si on les remet quelque part, ça change pas.

$\mathrm{E}:$ Ben on les détruit.

Et comment tu détruis des sédiments ?

Long silence

Qu'est-ce qu'on pourrait faire d'autre?

Long silence

A : Ben à part arrêter de mettre des produits chimiques, je vois pas.

Et comment tu veux, comment tu ferais pour arrêter?

E : Ben mettre des lois.

Interdire ?

E : Oui, ça déjà ça limiterait.

Et toi, qu'est-ce que tu en penses?

A : Ben je sais pas trop parce que ben on a quand même besoin des produits chimiques pour construire, et après ben on saurait pas où les rejeter donc... je sais pas où ça pourrait aller.

Donc là, y'a une contradiction... 
A : Oui, on en a besoin, mais euh, il faut pas [...]

Dans l'extrait ci-dessus, les prises de parole sont prudentes et exclusivement en réponse aux questions de l'adulte, lequel assume seul l'avancement du raisonnement et la mise en lumière des contradictions.

\subsection{3. " Auteurs » potentiels}

Ceci contraste avec le discours des élèves qui questionnent et discutent d'eux-mêmes les intérêts et limites, la cohérence ou la pertinence des connaissances mobilisées dans les documents supports de l'entretien. L'extrait suivant est un exemple de ce mode d'appréhension des enjeux de durabilité caractéristique de l'ensemble en bas à droite de la figure 4 .

[...] Que pensez-vous de ces nouvelles informations?

V : Déjà, si c'est seulement sur un site

Déjà, pardon?

V : Si c'est seulement sur un site web... Si toutes ces informations sont sur un seul site web, j'aurais déjà fini mon diapo avec trois ou quatre sites web.

D'accord

$\mathrm{V}$ : Et puis, surtout, y'a des informations extrêmement intéressantes, telles que l'impact qu'a les polluants sur les espèces, en fait.

$\mathrm{Mm}$

$\mathrm{V}:$ Vu que... au niveau des sites même officiels, il n'y a que très très peu d'informations sur l'impact sur les espèces

Concernant la Seine tu veux dire?

V : Oui, ou les points d'eau alentours. Telle que par exemple la petite rivière qui est à côté.

G : Déjà sur le Saint-Laurent, je trouve qu'ils ont plus d'informations que nous sur la Seine, et que c'est pas mal de savoir un peu tout ça sur le Saint-Laurent et... et voilà.

V : Moi, de mon côté, y'a limite autant d'informations sur ce site web que ce que j'ai réussi à trouver en quasiment une année sur tous les sites web sur j'ai cherché.

Ok. Et donc le document il dit que la pollution chimique du Saint-Laurent a changé. Vous avez vu ça ? Vous avez pu lire ça ? Est-ce que cela vous interpelle ? Pourquoi elle a changé ? Estce que vous pouvez revenir un peu là-dessus, et peut être faire le lien avec ce qu'il se passe... ici pour la Seine ? Est-ce qu'on voit les mêmes problématiques ici ? Est-ce que ça vaut pour tous les fleuves?

$\mathrm{V}$ : Et bien c'est vrai, la pollution a changé du au fait que ont retiré, ils ont retiré des produits extrêmement néfastes. Mais ils ont trouvé des nouveaux, donc... est-ce qu'on peut dire que la pollution a changé ? Peut-être qu'on trouve d'autres types de pollution. 
C'est ça, oui

G : Ils ont enlevé des produits chimiques de l'agriculture etc, pour les fleuves - même pour les animaux, parce qu'il y avait des animaux qui étaient intoxiqués par les produits chimiques dans les champs - et c'est vrai que ça en soi, c'est pas mal. Ça a fait une évolution... mais après c'est vrai que, comme dit V, il y a des nouveaux produits chimiques. Alors est-ce que ça a changé avec les nouveaux produits chimiques ou c'est une évolution euh... voilà, s'il y a moins de pollution, quoi.

$\mathrm{V}$ : Est-ce que ces produits chimiques, finalement, ils étaient là depuis longtemps ? Et donc finalement, la pollution a diminué mais on trouve de nouvelles facettes ?

Oui

$\mathrm{V}$ : Et puis si on interdit un produit, il y a un risque que les industriels en trouvent un nouveau qui peut être tout aussi - voire même plus - néfaste que l'ancien.

D'accord. Et il faut un peu de temps pour s'en rendre compte, des impacts. Oui, OK

$\mathrm{V}$ : Le problème, c'est qu'en fait, on a plutôt tendance à interdire des produits plutôt qu'à en autoriser.

C'est-à-dire ?

$\mathrm{V}$ : C'est-à-dire que finalement, le problème c'est que... on part du principe que on autorise tous les produits, et que on interdit ceux qu'on a trouvé néfaste.

$\mathrm{Mm}$

Mais le problème, c'est qu'on ne sait pas s'ils sont... on a pas testé... on peut pas tester tous les produits en un seul coup.

$\mathrm{OK}, \mathrm{Mm}$

C'est mieux de tester un produit et après, l'autoriser. [...]

L'analyse par modalités montre que les 11 élèves qui questionnent en particulier la fiabilité des informations qu'ils reçoivent ont aussi une bonne autonomie de problématisation et ont su prendre en charge la complexité, mais davantage à l'échelle locale. Sur le plan cognitif, leur approche privilégie la dimension épistémique des rapports aux savoirs. On peut toutefois souligner deux éléments remarquables : d'une part ces élèves proposant des solutions créatives ne constituent que $19,6 \%$ de l'échantillon, d'autre part leur prise en charge de la complexité globale n'a pas été significative (sans quoi le lien avec cette variable aurait été repéré par l'analyse par modalités). Ils sont donc potentiellement en dynamique «d'auteurs », mais cette dynamique reste partielle et son développement pourrait être accompagné dans des situations didactiques spécifiques.

Il ressort de ces analyses le besoin de penser des situations d'enseignement/apprentissage adaptées aux approches de ces différents profils, ne reconnaissant pas seulement la dimension épistémique des savoirs mais prenant aussi en considération les types d'engagements exprimés pour favoriser l'intégration des contextes locaux dans une prise en compte globale de la complexité des enjeux planétaires de durabilité. 


\section{Conclusions et perspectives}

Nous avons effectué dans cette étude le croisement entre la typologie de l'engagement des jeunes en milieu scolaire, le rapport aux savoirs et au territoire et les caractéristiques des raisonnements socio-scientifiques (RSS) mis en œuvre à propos des enjeux de durabilité de leur territoire de proximité confronté à celui d'autres environnements géo-socio-culturels. Les résultats obtenus par une méthode mixte de recueil de données et d'analyses conduisent à dégager les points forts suivant :

- le type d'engagement de ces jeunes est fortement corrélé aux caractéristiques géo-sociologiques des établissements et ce en lien avec CSP parentaux et notamment maternels, ce qui est conforme avec les études génériques menées sur l'engagement citoyen des jeunes ;

- les élèves qui sont les moins autonomes dans la problématisation des enjeux de développement durable de leur territoire de proximité sont ceux qui ont peu d'investissement identitaire et qui interrogent peu les normes sociales. Qualifiables de scolaires, ils ont une attitude de doute ou de spectateur réflexif en attente d'évènements cognitifs, voire culturels, déclencheurs de dynamiques nouvelles ;

- les élèves pour lesquels la composante identitaire et sociale de leur rapport aux savoirs est fortement exprimée estiment être démunis pour penser les questions environnementales et de développement (QED), et s'engager sur ces questions alors que leur potentiel de critique politique des normes sociales est remarquable. Qualifiés de «paradoxaux », ils reportent sur les autorités la prise en charge de ces questions ;

- la qualité de la composante épistémique du rapport aux savoirs et des RSS que ces jeunes mobilisent constitue un levier indispensable pour penser les enjeux de durabilité territoriaux locaux et pour conduire à des dynamiques d'élèves auteurs dans leur engagement sur les QED.

L'ensemble de ces résultats nous conduisent à proposer le modèle suivant (Figure 5) :

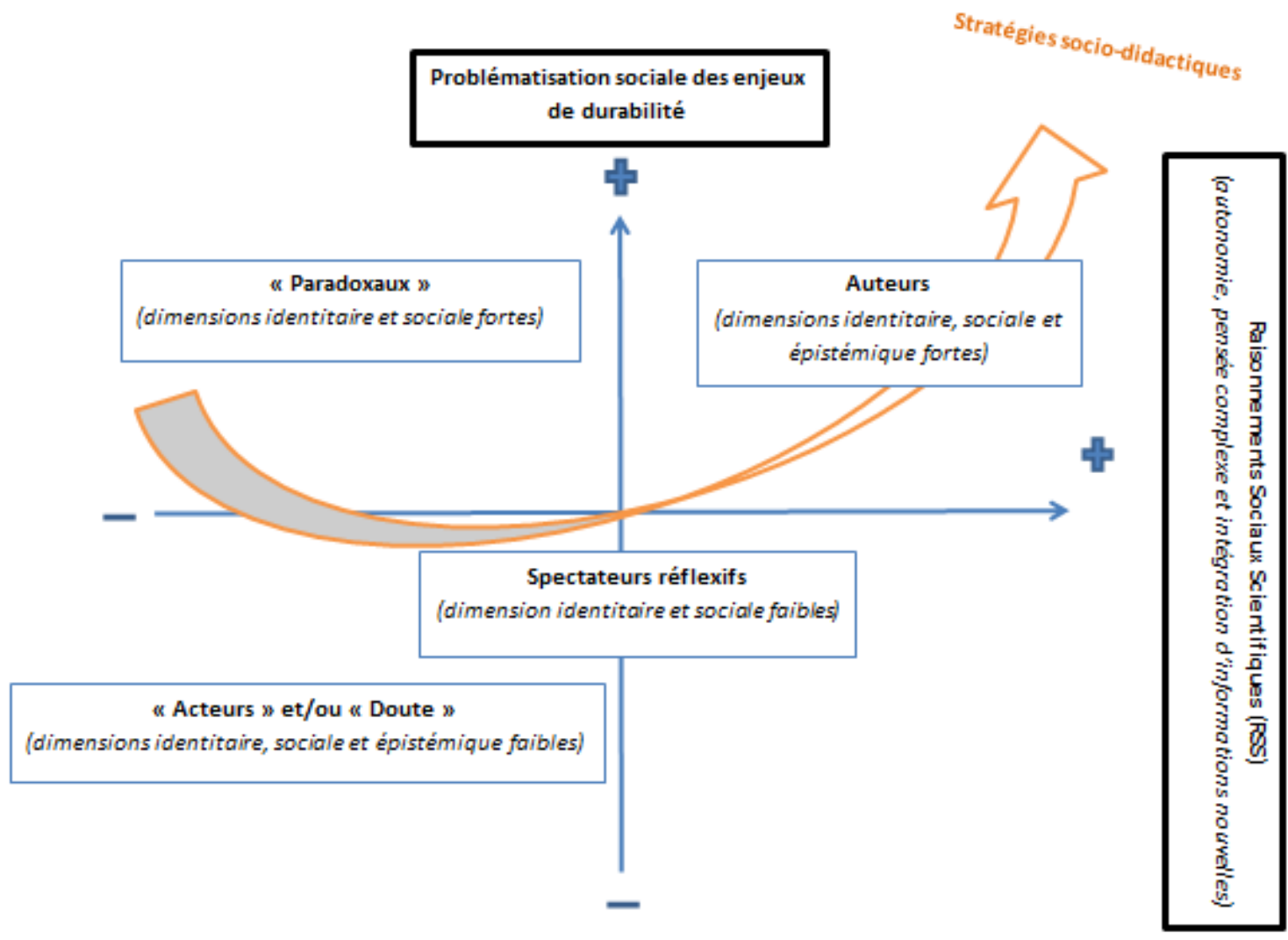

Figure 5. Dynamiques d'engagement relatives aux QED territoriales et caractéristiques cognitives de jeunes scolarisés 
Les caractérisations ainsi effectuées permettent d'envisager des stratégies socio-didactiques contextualisées plus efficientes au moyen des leviers de la critique des normes sociales aux différentes échelles d'appartenances, et du développement d'une pensée complexe et autonome mobilisant des savoirs robustes, ancrés dans des disciplines contributives.

\section{Bibliographie}

Bader, B., Horman, J. et Lapointe, C. (2010). Fostering Community and Civic Engagement in Low Income Multicultural Schools. Exceptional Education International, 20(2), 25-37.

Bader, B., Morin, E., Therriault, G. et Arseneau, I. (2014). Rapports aux savoirs scientifiques et engagement écocitoyen d'élèves de quatrième secondaire face aux changements climatiques. Revue francophone du développement durable, 4, 171-190.

Bandura, A. (1986). Social foundations of thought and action: A social cognitive theory. Englewood Cliffs: NJ: Prentice-Hall.

Barthes, A. (2018, March). The Hidden curriculum of sustainable development: the case of curriculum analysis in France. Journal of Sustainability Education, 18. Récupéré sur http://www.susted.org/

Benzecri, J. (1973). L'analyse des données, tome 2 : L'analyse des correspondances. Paris: Dunod.

Bugnard, P.-P., Freudiger, N., Haeberli, P., \& Pache, A. (2009). Débats en classe, entretiens d'élèves et savoirs dans l'éducation au développement durable. Dans Symposium international Ecole(s) et culture(s) : Quels savoirs ? Quelles pratiques ? Lille, 12 et 13 novembre: Université Lille 3.

Charlot, B. (1997). Du rapport au savoir, éléments pour une théorie. Paris: Anthropos.

Fabre, M. (2017). Problématisation-Problèmes flous. Dans A. Barthes, J.-M. Lange, \& N. Tutiaux-Guillon, Dictionnaire critique des enjeux et concepts des "éducations à" (pp. 216-218). Paris: L'harmattan.

Jenkins, EW, G Pell, G. (2006). The Relevance of Science Education Project (ROSE) in England: a summary of findings. Leeds.ac.uk. http://www.leeds.ac.uk/educol/documents/152736.htm

Kalali, F. (2017). Rapport à. Dans A. Barthes, J.-M. Lange, \& N. Tutiaux-Guillon, Dictionnaire critique des enjeux et concepts des "éducations à" (pp. 544-547). Paris: L'harmattan.

Kitzinger, J., Marková, I., \& Kalampalikis, N. (2004). Qu'est-ce que les focus groups ? Bulletin de psychologie, 57(3), 237-243.

Lange, J.-M. (2012). Education in sustainable development: how can science education contribute to the perception of vulnerability? Research In Science Education, 42, 109-127.

Lange, J.-M. (2014). Des dispositions des personnes aux compétences favorables à un développement durable : place et rôle de l'éducation. Dans A. Diemer, Éducation au développement durable Enjeux et controverses (pp. 163-182). Louvain-La-Neuve: De Boeck supérieur.

Lange, J-M. et Barthes, A. (2020). Déterminants de l'engagement de jeunes en fin de scolarité obligatoire vis-à-vis des enjeux de durabilité/soutenabilité. Dans M. Barroca-Paccard et S. Demers « Crise écologique: citoyennetés en lutte et éducation ». Numéro spécial EducationS, ISTE, London.

Lange, J.-M., \& Martinant, J.-L. (2010). Education au développement durable et éducation scientifique : Repères pour un curriculum. Dans A. Hasni, \& J. Lebeaume, Enjeux contemporains de l'éducation scientifique et technique (pp. 125-154). Ottawa: Les Presses de l'Université d'Ottawa.

Lange, J.-M., Janner, M., \& Victor, P. (2011). Assess efficiency of education for sustainable development in obligatory school: Toward relevant indicators. Dans Conference of the European Science Education Research Association (ESERA). Lyon: 7- 9 septembre 2011.

Lange, J.-M., Trouvé, A., \& Victor, P. (2007). Expression d'une opinion raisonnée dans les éducation à... : quels indicateurs ? Strasbourg Actes du congrès international de l'Actualité de la Recherche en Education et en Formation AECSE. 29 aout-1 septembre.

Larini, M., \& Barthes, A. (2018). Statistiques et traitement de données quantitatives en éducation. London: ISTE editions

Levinson, R. (2010). Science education and democratic participation: an uneasy congruence? . Studies in Science Education, Vol.46 ( $\left.{ }^{\circ} 1\right)$, 69-119. 
Marková, I. (2003). Les focus groups. Dans S. Moscovici, \& F. Buschini, Les Méthodes des sciences humaines (pp. 221-242). Paris: Presses Universitaires de France.

Morin, O. (2013). Éducation à la citoyenneté et construction collaborative de Raisonnements SocioScientifiques dans la perspective de Durabilité : pédagogie numérique pour une approche interculturelle de Questions Socialement Vives Environnementales. Toulouse: Thèse de doctorat de l'Université Toulouse 2 Le Mirail.

Morin, O. (2017). Complexité et "éducations à". Dans A. Barthes, J.-M. Lange, \& N. Tutiaux-Guillon, Dictionnaire critique des enjeux et concepts des éducations à... (pp. 65-73). Paris: L'harmattan.

Zeyer, A. et Kelsey, E. (2013). Environmental Education in a Cultural Context. In R.B. Stevenson, M. Brody, J. Dillon et A.E.J. Wals (eds.), International Handbook of Research on Environmental Education. New York: Routledge. 
Les principales étapes de la démarche :

- Se présenter et présenter les lieux que l'on fréquente à d'autres élèves dans la plateforme EdModo

- Choisir un enjeu lié au Fleuve qui vous intéresse et justifier sa pertinence sociale et environnementale.

- Échanger sur votre intérêt concernant cet enjeu avec un.e chercheur.e

- Documenter cet enjeu de manière interdisciplinaire en mettant à profit les différentes activités du projet (cours, conférences, visites, recherches, discussions, etc.).

- Échanger avec d'autres équipes dans la plateforme EdModo et enrichir la représentation de l'enjeu en vue de passer à l'action.

- Envisager et initier un scénario d'action pertinent lié à cet enjeu.

- Faire le point sur l'ensemble de la démarche avec un.e chercheur.e.

\section{Annexe $n^{\circ}$ 2. Guide d'entretien, première partie, autour de la demarche éducative}

Durant l'année scolaire qui s'achève, vous avez réalisé une démarche éducative entourant le développement durable de la Seine.

1. De manière générale, qu'est-ce que vous retenez d'intéressant de cette démarche ? Et que retenez-vous de moins intéressant ? Expliquez pourquoi.

2. Concernant la démarche dans son ensemble, ressemble-t-elle à une manière de faire à laquelle vous êtes habitués à l'école ? Quels aspects étaient différents et/ou semblables ? Précisez

3. Il y avait plusieurs étapes à la démarche que vous avez réalisée (les travaux, mais aussi la démarche de recherche documentaire, les sorties, etc.). Quel a été votre intérêt pour chacune de ces étapes ? Votre intérêt pour la démarche a-t-il évolué en cours d'année ? Expliquez.

4. Cette démarche vous a-t-elle permis de réaliser certains apprentissages ? Si oui lesquels ?

5. Particulièrement en lien avec l'enjeu lié au fleuve que vous avez documenté, que retenez-vous ? Qu'avez-vous appris d'étonnant, d'intéressant, d'important ou d'utile?

6. On vous a demandé d'imaginer un scénario d'action en lien avec le développement durable de la Seine. Pouvez-vous nous en parler un peu ? Comment l'avez-vous imaginé ? Si vous vouliez le réaliser, comment vous y prendriez-vous?

7. Avez-vous eu l'occasion d'échanger avec les autres élèves de votre classe ? Si oui, pourriez-vous nous dire ce que vous ont apporté les projets des autres groupes?

8. Vous avez été invité à échanger avec des élèves québécois via la plateforme Edmodo, mais peu d'échanges ont été réalisés. Pouvez-vous identifier des raisons qui ont freiné ou empêché ces échanges? 

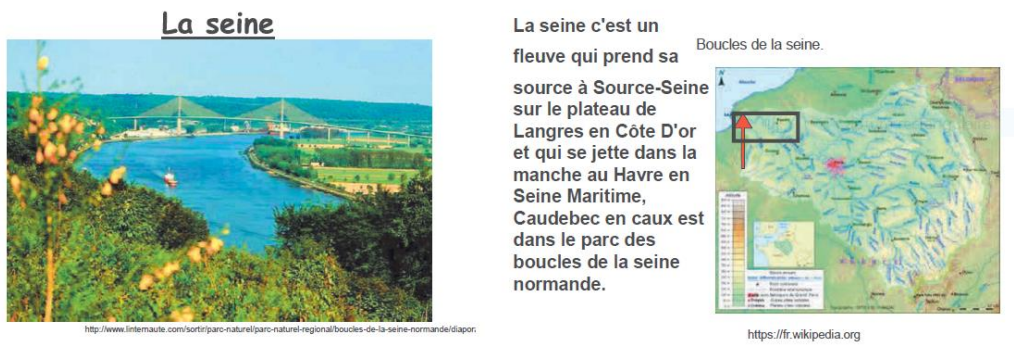

On trouve 32 espèces dans la seine ablette, anguille, barbeau, bouvière, brème, brochet, carassin, carpe, chabot, chevesne, épinoche, gardon, goujon, grémille, hotu, ide, loche, perche, poisson-chat, pseudorasbora, rotengle, sandre, silure glane, tanche, truite, vandoise.

Comment empêchons-nous que les population d'animaux de la seine décline avec le réchauffement climatique et la pollution?

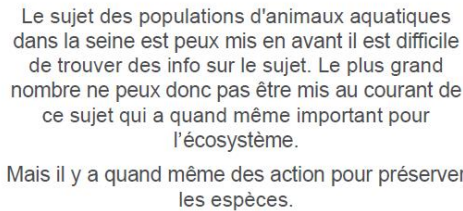

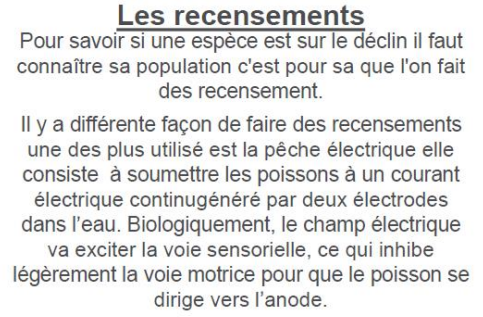

Mais est-ce que ces espèces sont en danger.

Au finale les espèces de la seine et des plans d'eau au alentour se porte plutôt bien mais il faut quand même faire des effort en lutant contre les espèces envahissante par exemple et en évitant de détruire les habitat

\section{Annexe n 4. Document 2 : "Voici un site qui traite de l'état du Saint-Laurent, des facteurs qui lui sont nuisibles ainsi que des lois actuellement en vigueur afin d'éviter la dégradation de cet important cours d'eau. " recommandation d'un élève de Québec le 9 avril 2019 dans la plateforme Edmodo}

https://baleinesendirect.org/des-baleines-en-peril/menaces/etat-de-sante-du-saint-laurent/

Depuis plus de deux siècles, le Saint-Laurent a subi de nombreuses avaries liées aux activités industrielles, à l'agriculture, à l'urbanisation ou au trafic maritime. Les changements climatiques déjà observables ajoutent une nouvelle donne. Le sujet est complexe et l'analyse requiert de la prudence, le travail des chercheurs étant toujours en cours. Voici un portrait de l'état de santé du Saint-Laurent.

\section{La pollution chimique change de nature}

Dans les années 1980 et 1990, les préoccupations principales concernaient des substances comme le plomb, le mercure et le cadmium ainsi que des molécules organiques telles que les HAP, DDT (insecticide), BPC et mirex (pesticide). Elles provenaient surtout des industries papetières, de l'aluminium et de l'agriculture. Dans le cadre du Plan d'action Saint-Laurent Vision 2000, plus de 100 usines situées en bordure du Saint-Laurent ont diminué leurs effluents toxiques de $90 \%$ depuis 1988. De bonnes nouvelles comme celles-ci ont des conséquences positives sur l'état de santé du Saint-Laurent, mais la guerre contre la pollution est loin d'être gagnée.

$[\ldots]$

Même si l'apport des toxiques a cessé ou considérablement diminué, ils sont encore présents dans le système; ils se sont déposés sur le fond et peu à peu intégrés dans les sédiments. Ils sont toutefois régulièrement remis en suspension, par le brassage naturel des eaux ou par le dragage nécessaire à l'entretien et au développement de la voie maritime du Saint-Laurent. Le TBT, peinture toxique anti-salissure, aujourd'hui interdit et retiré du marché, est encore sur la coque de beaucoup de navires commerciaux. Ce produit est hautement toxique pour plusieurs espèces aquatiques, incluant les mammifères marins. Les organobromés sont parmi les plus récentes substances dont la toxicité a été découverte 
bien après leur déploiement massif dans l'environnement. Ces produits, utilisés pour améliorer la résistance des matières plastiques au feu, sont présents dans une multitude de produits courants : boîtiers d'ordinateurs et de téléviseurs, composants électriques et électroniques, tissus, matériaux de rembourrage, etc. Si on connaît encore peu leurs effets sur l'environnement, on sait déjà que certains sont très persistants et en augmentation spectaculaire dans les organismes vivants : chez les bélugas du Saint-Laurent, les concentrations augmentent de façon exponentielle, doublant tous les trois ans. Une réglementation pour restreindre, voire interdire, leur usage est actuellement en développement.

Les égouts des municipalités et les eaux usées des bateaux représentent également une source de pollution. Selon une étude récemment parue, les mammifères marins sembleraient être de plus en plus victimes de maladies frappant habituellement les animaux domestiques, possiblement lié à ces transferts d'eau. Sur les deux rives du fleuve, la qualité des systèmes d'épuration est très inégale, et parfois sommaire. Toutes ces problématiques sont complexes, les réglementations évoluent et leur application prend du temps.

\section{Annexe $n^{\circ}$ 5. Carte mentale représentant les items et liens pris en compte dans le raisonnement des groupes dans leur production et lors du focus group.}

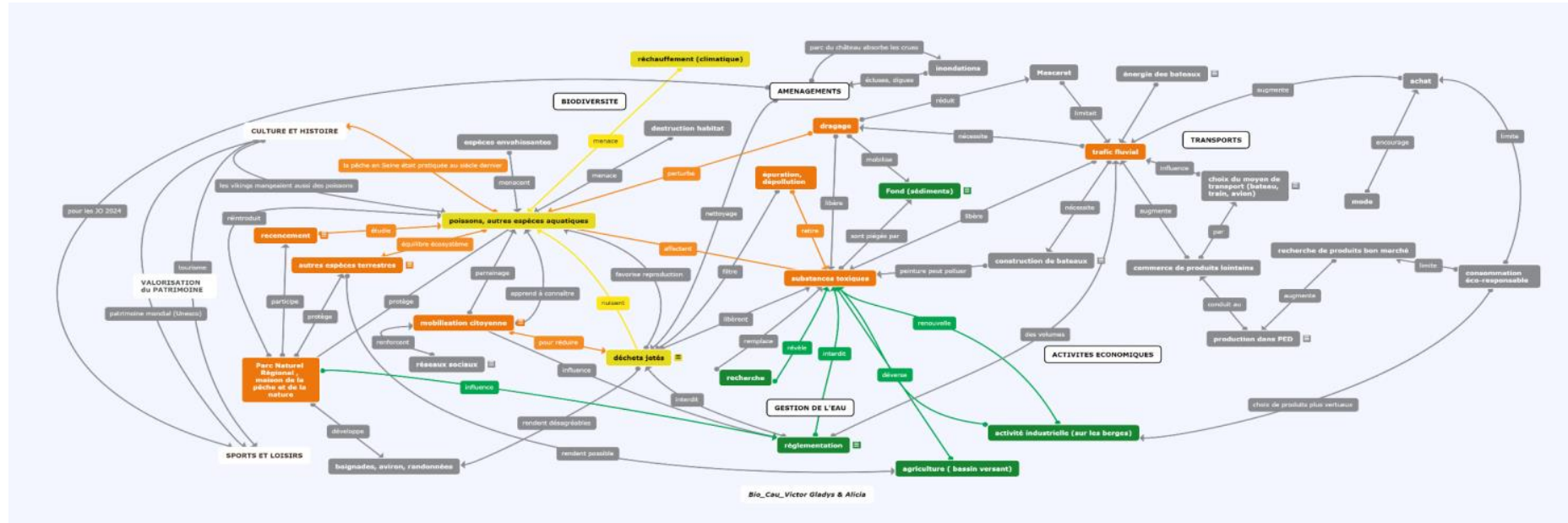

L'ensemble des items et des liens identifiés par l'ensemble des groupes constitue le réseau d'arrièreplan en gris. Pour le groupe étudié dans cet exemple, le rapport $11 / \mathrm{n} 1=2 / 3$ (éléments en jaune), $12 / \mathrm{n} 2=9 / 11$ (éléments en jaune et en orange), et 13/n3=15/16 (éléments en jaune, en orange et en vert) 